\title{
Paramedic direct referrals to senior decision maker: is this the way forward? A pilot project in acute general medicine and ambulatory care at the John Radcliffe Hospital, Oxford
}

\author{
Authors: Leila Vaziri, ${ }^{*}$ Mridula Rajwani, Jordan Bowen, James Price, Sudhir Singh, Anuja Bambaravanage* and \\ Megan Stewart
}

\begin{abstract}
Background
The Royal College of Physicians' 2013 Future Hospital report recommends ambulatory care 'by default' as a model to meet the increasing demands on acute services. ${ }^{1}$ The Oxford University Hospitals ambulatory assessment unit (AAU) was established in 2016 to provide an ambulatory alternative for patients referred to the undifferentiated medical take. A phone cascade system between $8 \mathrm{am}$ and $6 \mathrm{pm}$ enabled senior decision makers (consultant, chief registrars) to receive all initial calls about medical admissions from referring general practitioners as well as from paramedics and other healthcare providers. Despite the provision of a direct line to a senior clinician the majority of paramedic conveyances were to the emergency department (ED), including a proportion of patients eligible for redirection to the AAU.
\end{abstract}

\section{Aims}

To decongest the ED by directing an increased number of appropriate paramedic referrals to the AAU or the medical admissions unit.

\section{Methods}

> A collaborative pilot project was developed by engaging the senior trust leadership, the regional ambulance service and the local clinical commissioning group (CCG).

> Initial observational data collection of patients from ED triage conveyed by ambulance and subsequently seen in the ED.

> Discussion with the ambulance service leadership to identify barriers to referral and encourage calls of medical presentations directly to the medical referral phone.

$>$ Phone holder availability to referring paramedics increased to 24 hours.

> Educating medical registrars around ambulatory pathways in the region.

Authors: Oxford University Hospital

${ }^{*} \mathrm{RCP}$ chief registrar

\section{Results}

> Data collected on a single day in ED triage showed 2/43 (4\%) of paramedic referrals were seen on $\mathrm{AAU}$, with no patients referred directly to take. On review of the clinical presentations, 16\% could have been seen on AAU directly, and $25 \%$ could have been seen directly on the medical admissions unit.

> Pre-intervention $12 \%$ of calls made to the referral phone were from paramedic team, and post intervention that rose to $26 \%$ of total calls taken.

> Over a 1-week period during the pilot $73 \%$ of calls, from paramedics were directed to AAU or medical take, and therefore away from the ED. Only $17 \%$ were seen in ED. $10 \%$ were directed to other services, including specialties.

> The data also suggested that the AAU South Central ambulance service patients rose from $3.8 \%$ to $13 \%$, which has been maintained post the pilot week.

\section{Conclusion}

This pilot project sought to reduce the number of ambulance conveyances to the ED by removing barriers to paramedics accessing alternative pathways. Critical to this was enhancing an existing phone-cascade model providing a direct line for paramedics to discuss patients with senior decision-makers. In conjunction with an education programme and crossorganisational collaboration the number of discussed patients conveyed to ED was reduced by $73 \%$.

Although increasing the availability of senior decision support to non-medical referrers 'upstream' to acute hospital services may reduce ED conveyances, further work is necessary to understand the opportunity, cost, economics and professional barriers to widespread adoption.

\section{Reference}

1 Royal College of Physicians. Future hospital: Caring for medical patients. London: RCP, 2013. 